\title{
Construction of New Mechanism Based on Additive Constraint Kinematic Pair
}

\author{
Qu Zhigang \\ School of Mechanical Engineering \\ Harbin University Of Science And Technology \\ Harbin, China \\ e-mail: aaj800@sina.com
}

\begin{abstract}
In order to accomplish the real-time transfer of mechanism configuration during moving process, a new kinematic pair, named as additional constraint kinematic pair, is proposed. Being different from the traditional kinematic pair, this new kinematic pair can supply varying constraint number under certain additional conditions. Different kinds of additional constraint kinematic pair are listed in present study. Meanwhile, their structure styles and functions are also analyzed. Furthermore, by using the kinematic pair isomeric transforming principle and taking the double-slider five-bar stepping mechanism as the original structure, a new mechanical stepping mechanism is developed based on the additive constraint kinematic pair and its application is also examined. This additive constraint kinematic pair has guiding significance for innovative design of mechanisms (construction of new mechanisms), it has broad application prospect in automatic, feeding device, robot and toy, which has the major limitation is whether the mechanism structure of kinematic pair can work reliably or not.
\end{abstract}

Keywords-mechanism; additive constraint; kinematic pair; configuration; stepping

\section{INTRODUCTION}

Nowadays fixed structure mechanism made up of fixed constraint kinematic pair is most used. Comparing with fixed constraint, there is another kinematic pair which is called additive constraint kinematic pair and can provide variable constraint in additive conditions.

Creeping is an intermittent motion. An intermittent motion is a motion when driving parts rotate continuously, driven part move intermittently. Pause is the main feature of intermittent motion and non-motion state of element of a mechanism. Mechanical creeping machine is a intermittent motion mechanism which has configuration transforming function developed based on transforming factor principle. It has broad application prospect in automatic, feeding device, robot and toy[1-6].

\section{ADDITIVE CONSTRAINT KINEMATIC PAIR}

\section{A. Additive constraint kinematic pair}

Additive constraint kinematic pair is formed by applying some motion, force and geometry constraints to traditional fixed constraint kinematic pair. It includes three types:

1) limiting direction kinematic pair: additive constraints limit relative motion direction of two kinematic pair elements.

2) limiting position kinematic pair:

additive constraints limit relative motion scope of two kinematic pair elements.

3) floating connected to ground kinematic pair:

under some additive conditions the pair can move relatively to ground.

\section{B. Function and application of additive constraint kinematic pair}

Additive constraint kinematic pair will become immobile temporarily acted by additive constraints and it is called passive kinematic pair. Additive constraint kinematic pair can switch to-and-fro from traditional kinematic pair to additive constraint kinematic pair automatically in given conditions. The essential of transform is two components connected with one lower pair are replaced by one component. Additive constraint kinematic pair which make mechanism has many configurations is the key element of transforming mechanism .

\section{Configuration transforming principle of additive constraint kinematic pair}

The principle is a theory which introduce transforming factor additive constraint kinematic pair into primary mechanism and control constraint number by transforming factor to transform configuration of mechanism automatically. It includes two contents:

1) Selection and combination of primary mechanism and transforming factor

The aim is to achieve the expansion and combination of primary mechanism function through transforming factor.

2) automatic control of transforming factor constrain number

The main control method is geometry control, force control and motion state control, etc.

\section{Mechanism Configuration Transforming Factor--- Limiting Direction Kinematic Pairs}

Conception of mechanism configuration transforming factor is proposed. Mechanism configuration transforming factor which is shorten as transforming factor is an structural element in transforming mechanism. Mechanism configuration can be automatically 
transformed to achieve sequence technical operation by controlling constraint number provided by transforming factor. A mechanism with transforming factor has special function and is called transforming mechanism.

Transforming factors constructing transforming mechanism have many types. Limiting direction kinematic pair is a kind of transforming factor(shown in Fig .1a,b).
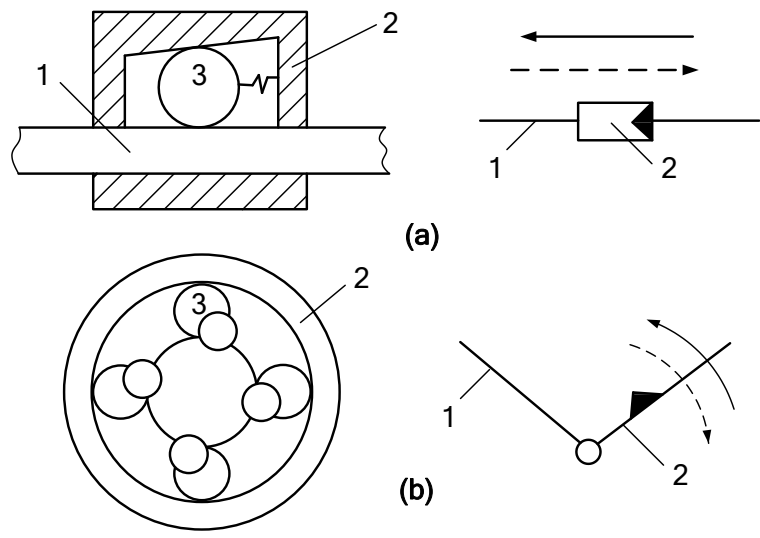

(a)

(b)

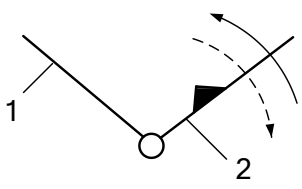

Figure 1. transforming factor ---limiting direction kinematic pair

\section{1) One-way prismatic pair}

One-way prismatic pair can only move one-way, as shown in Fig .1a. It can provide two constraints when moving along movable direction and provide three constraints when moving along immovable direction. It becomes immovable passive prismatic pair temporarily. Constraint numbers can automatically transform from 2 to 3 provided by one-way prismatic pair. When the pair is introduced into a mechanism, the mechanism is transformed to a transforming mechanism which has two configurations.

2) One-way revolute pair

One-way revolute pair can only revolute oneway(shown in Fig .1b). It can provide two constraints when rotating around ratory direction and provide three constraints when rotating around immovable direction. It becomes immovable passive revolute pair temporarily. Constraint numbers can automatically transform from 2 to 3 provided by one-way revolute pair. When the pair is introduced into a mechanism, the mechanism is transformed to a transforming mechanism which has two configurations.[7-9]

\section{E. Application of configuration transforming principle of additive constraint kinematic pair}

Fig .2 shows the structure diagram of armor plate cutting machine. Lower cutter frame 1, double eccentric shaft 2, pull rod 3, upper cutter frame 4 and frame 5 constitute the primary mechanism------double-slider fivebar mechanism whose DOF is two. The cutting machine must complete two continuous movements: compacting and cutting and hence transforming factor additive constraint prismatic pair(constructional element1frame) is inducted in the machine to accomplish reciprocal transformation between prismatic pair and passive prismatic pair under the control of additive conditions. passive prismatic pair is a motion state(selflock) of additive constraint kinematic pair. When additive constraint prismatic pair changes to passive prismatic pair, double-slider fivebar 2-DOF mechanism is transformed to fourbar 1-DOF mechanism----slider-crank mechanism and thus the machine can finish two continuous movements of compacting and cutting under definite motion conditions.

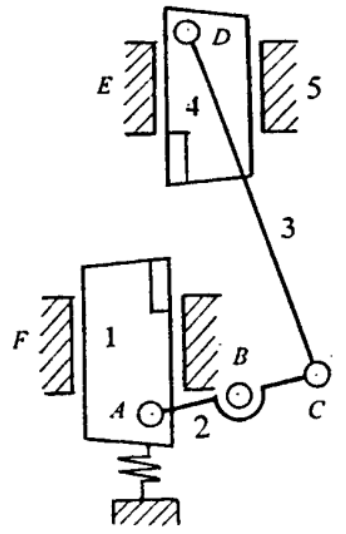

Figure 2. the structure diagram of armor plate cutting machine

\section{FORMING OF DOUBLE-SLIDER FIVE-BAR STEPPING MECHANISM WHICH IS THE ORIGINAL MECHANISM OF CREEPING MACHINE}

\section{A. Forming Of Double-Slider Five-Bar Stepping Mechanism}

Fig .3a shows a traditional double-slider five-bar mechanism which is composed of slider 1 , linkage 2 and 3 , slider 4 and frame 5 . Frame 5 provides slide rail for slider 1 and 4 to form two prismatic pairs. The two sliders are connected to the frame.

(a)

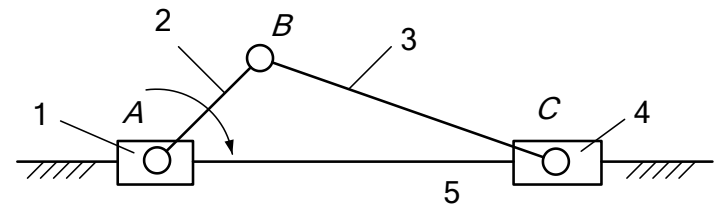

(b)

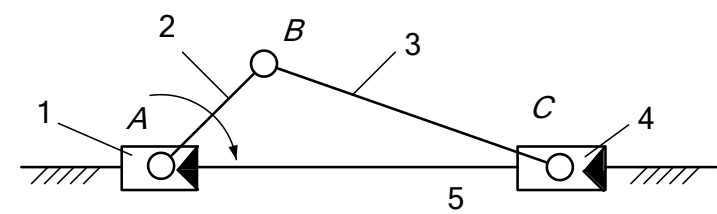

Figure 3. double-slider five-bar stepping mechanism and its original mechanism

The DOF of double-slider five-bar mechanism is 2 . So according the definite motion conditions of mechanism, the five-bar mechanism must has two driving parts to achieve definite motion. If it has only one driving part, e.g. we choose linkage 2 as the driving part, the five-bar mechanism can only move according to minimum resistance law. Driving part AB rotates clockwise around dot A with $\omega$ and passes the force to slider 4 through linkage 3. At the same time the pressing shaft force of $A B$ through the direction of $\mathrm{AB}$ linkage act on the slider 1. By 
the force analyse of slider 1 and 4 and contrasting it we can find the minimum resistance law of the mechanism. But the mechanism moving according to minimum resistance law can't stand load. Because the resistance will change as soon as the mechanism stand load and thus induce the changing of motion law. Hereby we can get the conclusion that loading-bearing mechanism must work under the definite motion conditions, that is, the numbers of driving parts must equal to DOF of the mechanism.

So a double-slider five-bar mechanism must be changed if it has definite motion with only one driving part. By using variable constraint kinematic pair principle and one-way prismatic pair technology, a double-slider five-bar mechanism is evolved into a double-slider fivebar stepping mechanism, as shown in Fig .3b.

The key technology of double-slider five-bar stepping mechanism is using a variable constraint kinematic pair--one-way prismatic pair. The function of the pair is that two connected components only move one-way and can't move another way.

\section{B. Stepping Motion Analyse}

We suppose the original position of stepping motion as $A B$ overlap coincident with BC (shown in Fig .4a). Slider A and B can only move leftwards and If they move rightwards, self-lock happens. We suppose $A B$ which isn't connected to the frame as the driving part and rotate clockwise.

The motion course of double-slider five-bar stepping mechanism can be described as follows: Driving part AB rotate clockwise from original position from 00 to 1800 . During the process, one-way prismatic kinematic pair slider C can't move leftwards because of the pressure of linkage $\mathrm{BC}$ and it remains standstill. At the same time $\mathrm{AB}$ drives $\mathrm{BC}$ rotate around point $\mathrm{C}$ and contrarily drives oneway prismatic kinematic pair slider A to move leftwards to point A1. Displacement is $2 \mathrm{LAB}$, as Fig . $4 \mathrm{~b}$ shows. The mechanism equals to a movement guide pole mechanism.

Driving part $\mathrm{AB}$ rotates continuously from 1800 to 3600. During the process, one-way prismatic kinematic pair slider A stops at point A1 and BC pulls one-way prismatic kinematic pair slider $\mathrm{C}$ to move leftwards. Displacement is $2 \mathrm{LAB}$, as Fig .4c shows. The mechanism equals to a slider crank mechanism.

It's a motion circle when $\mathrm{AB}$ rotates one-circle. During the circle, one-way prismatic kinematic pair slider A and C intermittently orderly move leftwards $2 \mathrm{LAB}$. Thus double slider stepping motion is formed and its gait is double foot shift walking.

(a)

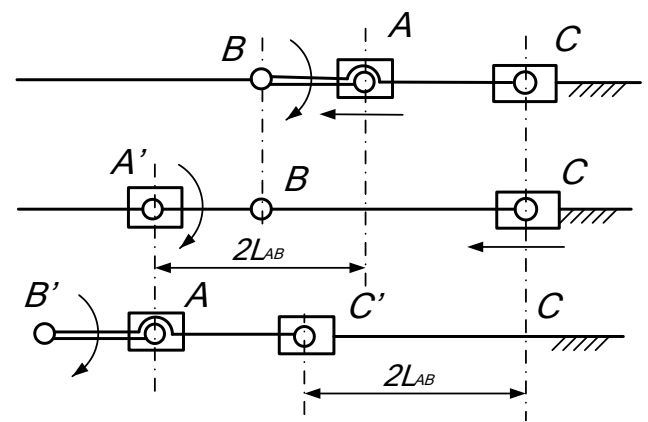

Figure 4. Motion of double slider five-bar stepping mechanism

\section{MECHANICAL CREEPING MACHINE}

The structure feature of double slider five-bar stepping mechanism is that transforming factor one-way prismatic pair is lead rail type and so transforming mechanism is also lead rail type. These limit their application range.

For the sake of enlarging application range of stepping mechanism based on transforming factor, we apply kinematic pair slide roll shift principle and introduce another transforming factor one-way revolute pair into lead rail type stepping machine to innovate its structure. A new transforming mechanism----railless walking machine which has more application range is developed.

By taking double slider five-bar stepping mechanism(shown in Fig .3) as original mechanism, we apply kinematic pair slide roll shift principle and isomeric evolving principle to innovate structurally slider A and slider B. That is, to find the isomer of one-way prismatic kinematic pair. Isomer means they have same feature--one-way move and different structures.

\section{A. Slide-Roll Shift Of Prismatic Pair}

The motion state of prismatic pairs in original mechanism(shown in Fig .3a )is slide. If we introduce a middle roller which has two kinematic pairs ( a revolute pair and a higher pair) into the two elements of prismatic pair, we get a combination prismatic kinematic pair(shown in Fig .5b and d) which is composed of roller, revolute pair and pure roll kinematic pair.

The one-way prismatic pairs in the original mechanism(shown in Fig .3b)are limiting direction kinematic pairs same to the pairs in Fig .1a. They can move leftwards and can't move rightwards. By applying isomeric principle we evolve the original mechanism structurally as follows: transfer the frame rail 5 to plane 5 , introduce limiting direction revolute pair (shown in Fig .1b) as the rollers of complex prismatic pair and make them rotate counter clockwise and can't rotate clockwise, make complex prismatic pair only move leftwards and can't move rightwards.

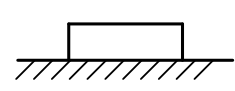

(a)

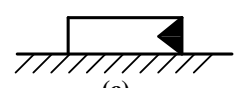

(c)

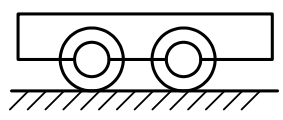

(b)

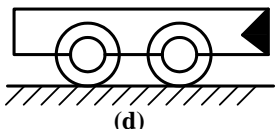

(d)
Figure 5. Generalized prismatic pair

A generalized prismatic pair is a relative movement connection between two components no matter what the structure of intermediate is.

\section{B. Mechanical creeping machine}

A mechanical creeping machine is formed by introducing one-way combination prismatic pair into 
double slider five-bar stepping mechanism to replace the two one-way prismatic pairs. The machine is composed of front wheel carrier 1 , crank 2, linkage 3, back wheel carrier 4 and plane frame 5 .

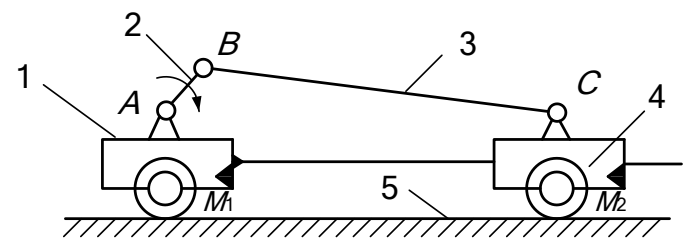

(a)

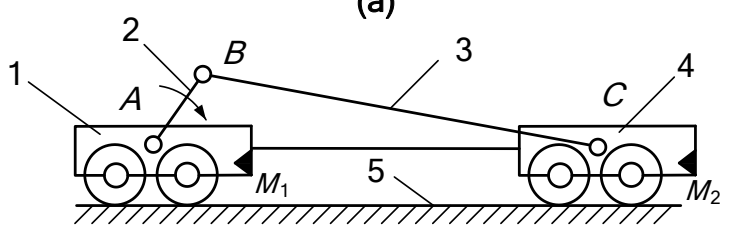

(b)

Figure 6. Mechanical creeping machine

In Fig .6a, wheel carrier M1 and M2 is connected by a prismatic pair to keep M1 and M2 parallel to the ground during the translation to form generalized prismatic pair. If the wheels of M1 and M2 are double, the carriers can keep parallel to the ground themselves and can move relatively. Thus the prismatic pair of $\mathrm{M} 1$ and $\mathrm{M} 2$ is thrown off and self-adaptive property of the creeping machine is enhanced, as shown in Fig .6b.

Geometrid is a sort of insect. It has thin body and legs, broad wings and compound eyes. The inchworm is the larva of geometrid and it has double creeping feet at the head and cauda. During creeping firstly back foot creeps forwards to hunch its body. Secondly back foot creeps forwards to stretch its body. Thus it does again and again. The walking process is just like we using a thumb and a middle finger to span, so it is called inchworm.

An inchworm model is made. Front-wheel carrier M1 and rear wheel carrier M2 of mechanical creeping machine are respectively packaged by fore leg and hind leg of inchworm. Using one-way alternant movement of front-wheel chassis M1 and rear wheel chassis M2 to imitate alternant creeping of fore and hind leg of inchworm to form mechanical bionic inchworm, as shown in Fig .7.[10-11]

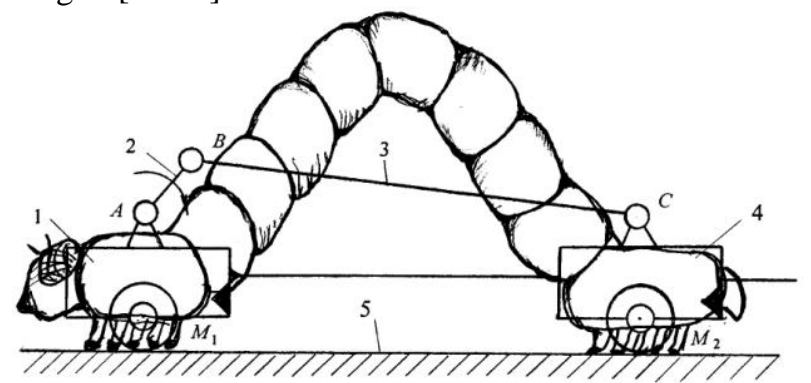

Figure 7. Mechanical bionic inchworm

\section{CONCLUSIONS}

Prismatic pair 4 is transformed to additive constraint prismatic pair by introducing force additive constrain in primary mechanism( double-slider fivebar mechanism).The pair can automatically transform from traditional prismatic pair to passive prismatic pair.

Transforming factor additive constraint kinematic pair is key element to construct transforming mechanism.

Mechanical configuration transforming factor---limiting direction kinematic pair is presented. Two configuration transforming factors----limiting direction prismatic kinematic pair and limiting direction kinematic pair are developed.

A rail double slider five-bar creeping machine is constructed based on transforming factor---limiting direction prismatic kinematic pair.

A limiting direction prismatic pair is evolved isomericly into a generalized prismatic pair using transforming factor---one-way revolute pair as the elements.

A railless mechanical creeping machine is developed based on transforming factor--- generalized prismatic pair. An application example---mechanical bionic inchworm is given.

\section{ACKNOWLEDGMENT}

This work supported by the Key Program of National Natural Science Foundation of Heilongjiang No.ZD201309, and Project supported by the Maor International Joint Research Program of China (Grant No. 2013DFA71120).

\section{REFERENCES}

[1] Huang Long; Yu Guangbin ETC.,Research on nonlinear vibration characteristics of non-orthogonal face gear transmission , Information Technology Journal, 2013(8),8102-8108

[2] Sun Yongguo, Zhao Xingfu, Yu Guangbin etc.Backlash analysis of RV reducer based on Error Factor Sensitivity and MonteCarlo Simulation, International Journal of Hybrid Information Tec hnology, 2014(6) ,283-291

[3] Guangbin Yu, Chaolong Tang, Jinhui Song, and Wenqiang Lu. Physical model construction for electrical anisotropy of single crystal zinc oxide micro/nanobelt using finite element method, applied physics letters, 2014,104(15)

[4] Li Lin, Zhang Jian. Analyses and Uses of Variable Freedom Mechanism. Journal of Shandong of Light Industry, 1994,8 (3):4245

[5] Ma Luzhong, Sheng Yu, Yuan Huishan. Analysis and Application of Indefinite Freedom Mechanism. MTM'97[C]: 78-82

[6] Dai Jiansheng,Jones J Rees. Mobility in Metamorphic Mechanism of Foldabic/Erectable Kinds[J]. Transaction of the ASME. Journal of Mechanical Design. 1999,121(3):375-382

[7] Li Duanling, Dai Jiansheng. Structure Synthesis of Metamorphic Mechanisms Based on the Configuration Transformations. Chinese Journal of Mechanical Engineering.2002, 38(7):12-16

[8] Qu Zhigang, Li guixian, An Zijun. Theory and Application of the Mechanism Conformation Changebeing Based on Transforming Factor Control. 2005,41(2): 41-45

[9] Dai Jiansheng. Characteristics of Metamorphic Mechanisms Machine Design and Research. 2004supplement:276-278

[10] Qu Zhigang, An Zijun .Study on the function and application of mechanism with changeable degree of freedom. Mechanical Transmission, 2001, 25 (2): 6 8

[11] Qu Jifang, Yi Yali, Qu Zhigang. Technology innovation. Metallurgy Industry Press, 2005:168 171 